\title{
Flexible Metallic Nanowires with Self-Adaptive Contacts to Semiconducting Transition-Metal Dichalcogenide Monolayers
}

Junhao Lin ${ }^{1,2}$, Ovidiu Cretu ${ }^{3}$, Wu Zhou ${ }^{2}$, Kazu Suenaga ${ }^{3}$, Dhiraj Prasai ${ }^{4}$, Kirill I. Bolotin ${ }^{1}$, Nguyen Thanh Cuong ${ }^{3}$, Minoru Otani ${ }^{3}$, Susumu Okada ${ }^{5}$, Andrew R. Lupini', Juan-Carlos Idrobo ${ }^{2}$, Dave Caudel $^{1,6}$, Arnold Burger ${ }^{6}$, Nirmal J. Ghimire ${ }^{2,7}$, Jiaqiang Yan ${ }^{2,8}$, David G. Mandrus ${ }^{2,7,8}$, Stephen J. Pennycook $^{2,8}$, Sokrates T. Pantelides ${ }^{1,2}$

1. Department of Physics and Astronomy, Vanderbilt University, Nashville, TN 37235, USA

2. Materials Science \& Technology Division, Oak Ridge National Laboratory, Oak Ridge, TN 37831, USA

3. National Institute of Advanced Industrial Science and Technology (AIST), Tsukuba 305-8565, Japan

4. Interdisciplinary Graduate Program in Materials Science, Vanderbilt University, Nashville, TN 37235 , USA

5. Graduate School of Pure and Applied Sciences, University of Tsukuba, Tsukuba 305-8571, Japan

6. Department of Physics, Fisk University, Nashville, TN, 37208, USA

7. Department of Physics and Astronomy, University of Tennessee, Knoxville, TN 37996, USA.

8. Department of Materials Science and Engineering, University of Tennessee, Knoxville, TN 37996, USA

Two-dimensional transition-metal dichalcogenide (TMDC) monolayers, most of which are semiconducting with direct bandgaps, are promising candidates for flexible nanoelectronics [1]. TMDCbased atomically-thin devices have inspired research on assembling them into circuits [2, 3]. As the TMDC-based devices scale down to tens of nanometers, flexible conducting wires with efficient and robust junctions are essential for connecting multiple components in a nano-circuit for flexible electronics applications.

Notably, one-dimensional ultrathin transition-metal chalcogenide nanowires have been predicted to be metallic. These nanowires could potentially serve as a better conducting interconnect for future TMDCbased flexible nanoelectronics since no extra elements are introduced, therefore, simplifying the fabrication process and providing reliable interconnections. Although such metallic nanowires have been synthesized individually, controllable connections of nanowires to the monolayers, an important step towards assembling them into devices, have so far remained elusive.

Here, we report direct electron-beam fabrication of such ultrathin nanowires, including their ramified junctions, connecting designated points within a semiconducting TMDC monolayer (Fig. 1). The controllable fabrication is performed in a scanning transmission electron microscope with $5^{\text {th }}$ order aberration corrector which simultaneously records the movements of the atoms during the fabrication. The sequential images reveal that the formation of the nanowires is a self-regulating and self-healing process that is insensitive to precise beam parameters. In-situ electrical measurements further reveal the nanowires are intrinsically metallic. The nanowires remain conducting and maintain structural integrity as they undergo continuous electron-beam-induced rotations and flexing, indicating their self-adaptive connections to the monolayers. The observed mechanical behavior is explained by density-functionaltheory calculations, which further predict that the metal-semiconductor contacts could be Ohmic to p- 
type TMDC monolayers. These sub-nm-wide metallic nanowires can, therefore, serve as the ultimately small interconnects in future flexible nano-circuits fabricated entirely within a monolayer. [4, 5]

\section{References:}

[1] Wang, Q.H., et al., Nature Nanotechnology, 2012. 7(11) 699

[2] Radisavljevic, B., et al., Acs Nano, 2011. 5(12) 9934

[3] Wang, H., et al., Nano Letters, 2012. 12(9) 4674

[4] Lin, J.H., et al., submitted.

[5] This research was supported in part by U.S. DOE grant DE-FG02-09ER46554 (JL, STP), a Wigner Fellowship through the Laboratory Directed Research and Development Program of Oak Ridge National Laboratory, managed by UT-Battelle, LLC, for the U. S. DOE (WZ), the Office of Basic Energy Sciences, Materials Sciences and Engineering Division, U.S. DOE (ARL, NJG, JQY, DGM, SJP, STP), and through a user project supported by ORNL's Center for Nanophase Materials Sciences (CNMS), which is sponsored by the Scientific User Facilities Division, Office of Basic Energy Sciences, U.S. DOE. KIB and DP were supported by ONR N000141310299. This research used resources of the National Energy Research Scientific Computing Center, which is supported by the Office of Science of the US Department of Energy under Contract No.DE-AC02-05CH11231. OC and KS thank the JST research acceleration program for financial support. NTC, MO and SO acknowledge the JST-CREST program.

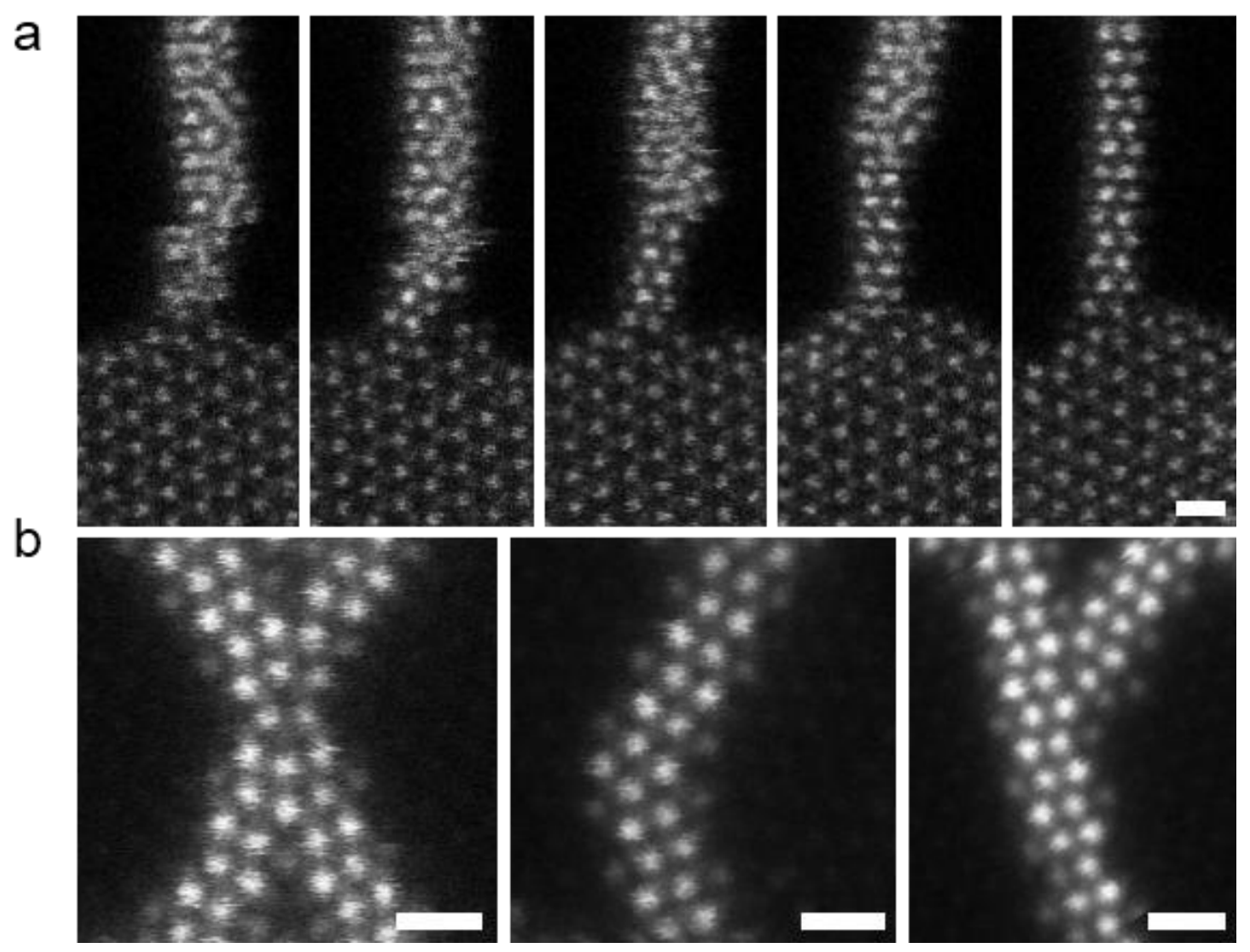

Figure 1: In-situ fabrication of nanowires from a TMDC monolayer and different types of junctions. (a) Sequential ADF images showing the fabrication process of an individual MoS nanowire entirely within a semiconducting $\mathrm{MoS}_{2}$ monolayer. (b) ADF images of different types of nanowires and ramified junctions created by advanced control of the electron irradiation inside the STEM, demonstrating the diversitv of building blocks made from these nanowires. Scale bars: $0.5 \mathrm{~nm}$. 